\title{
Nature-Based Physical Activity Advertising: Recommendations Based on Attention Restoration Theory and Psychoevolutionary Theory
}

\author{
Adam G. Gavarkovs \\ Faculty of Health Sciences, Western University \\ No conflicts of interest declared.
}

\begin{abstract}
Background Recent research has suggested that physical activity in natural areas may provide benefits that exceed those in other settings. Additional benefits include increased self-esteem and enjoyment of the activity, and decreased negative mood states and blood pressure. Therefore, encouraging nature-based physical activities may play an important role in the promotion of health and wellness. Advertising has been cited as an important component of a health promotion campaign; although to date no study has recommended strategies for designing advertising specific to nature-based physical activities. The purpose of this article is to review two prominent human-nature interaction frameworks, Attention Restoration Theory (ART) and Psychoevolutionary Theory (PET), and based on their tenets, recommend strategies for message design. The two recommendations proposed are: (1) to include natural images that meet the restorative criteria outlined in both theories; and (2) to explicitly feature the additional benefits of exercising in natural spaces in advertisements. Adhering to these recommendations in the advertisement design process may increase the effectiveness of the message.
\end{abstract}

\section{Introduction}

The lifestyles of Canadians are increasingly characterized by low physical activity levels. Only $15 \%$ of Canadian adults are currently meeting the recommended 150 minutes of moderate-to-vigorous physical activity per week (1). There is much evidence that suggests physical activity has a breadth of both physical and mental health benefits (2); for example, regular physical activity reduces the risk of both chronic diseases and mental health illnesses (2). Due to the potential role of physical activity in the prevention and treatment of medical conditions, continuing research in the promotion of physical activity is a public health priority.
One recent field of research in the area of physical activity promotion is the possible additional effects of physical activity in the natural environment. The term 'green exercise' was coined in 2003 and is defined as physical activity that takes place in natural environments (3). According to this definition, any type of physical activity (e.g., walking, running, mountain biking, golf, etc.) that takes place in outdoor settings such as woodlands, coastlines, beaches, urban parks, household gardens, and even tree-lined streets can be considered forms of green exercise (3).

One of the seminal explorations of the effects of green exercise versus physical activity in other settings was undertaken by Pretty and colleagues (4). In this study, five groups of 20 adult participants ran on a treadmill for 
20 minutes while viewing images of pleasant rural, unpleasant rural, pleasant urban, or unpleasant urban scenes, or no scenes at all. A variety of physiological and psychological outcomes, including blood pressure, mood, and self-esteem were then measured immediately following the bout of physical activity. Those who viewed pleasant rural scenes while exercising experienced the greatest decrease in blood pressure compared to those who viewed the other three scenes, or no scene at all. Viewing pleasant rural scenes also resulted in higher self-esteem and mood scores than running on a treadmill without viewing any scene, which by itself also produced positive outcomes. A subsequent study by Pretty and colleagues (5) also explored the impact of green exercise by administering a self-esteem and mood questionnaire to green exercise participants pre- and post-activity. A total of 263 participants, which included both children and adults $\left(M_{\text {age }}=47.8\right.$ years $)$, engaged in activities including walking, cycling, fishing, and horse riding, for a duration that varied from one to several hours. Compared to pre-activity scores, self-esteem improved significantly, and anger, confusion, depression, and anxiety all decreased significantly (5).

A recent systematic review conducted by Thompson Coon and colleagues (6) explored literature examining the benefits of green exercise versus exercising in other settings. A noteworthy finding of this review was that participating in nature based activity led to greater enjoyment and satisfaction with the activity and a greater intention to repeat the activity at a later date. In a similar review conducted by Gladwell and colleagues (7), other benefits of green exercise were discussed. Although the methodological rigour has been questioned in several of the studies that have explored this subject, evidence from these reviews has supported the general conclusion that the natural environment is a promising setting for physical activity promotion (6).

\section{Physical activity messaging}

Mass media messages are common components of physical activity promotion campaigns, and are occasionally used as the only component of public health efforts (8).
After conducting a systematic review of the literature exploring the effects of stand-alone mass media campaigns, the Community Preventative Services Task Force (affiliated with the Centers for Disease Control and Prevention) concluded that, considering modest results and methodological inconsistencies, they could not recommend these types of interventions as an effective way to promote physical activity (8). However, in a systematic review of the literature exploring the effects of community-wide physical activity campaigns which had mass media components, the Task Force concluded that, when featured as a component in a larger campaign, there was sufficient evidence to recommend mass media messaging as an effective strategy to promote physical activity (9). Based on these two large reviews of the literature, it is clear that mass media messaging plays an important role in the promotion of physical activity. However, messaging should be complemented by other health promotion activities (e.g., health education programs) to be optimally effective. Furthermore, as discussed in Brawley and Latimer (10), physical activity messages require careful development if they are expected to be successful. Messages that are not grounded in theory or are generic across different target audiences may be less than successful (10). As such, it is not only important that physical activity messages be included in the promotion process, but that these messages are carefully crafted.

Based on the empirical evidence of the unique benefits of physical activity in the natural environment, green exercise should be considered an important mechanism by which people can stay active, and one that deserves promotion. As highlighted above, a potentially important part of any physical activity promotional campaign (e.g., health education) is a mass media component. However, to the knowledge of the author, there are no studies that have proposed green-exercise-specific message design. Therefore, the purpose of this paper is to propose two recommendations for designing advertising materials specific to promoting green exercise. 
Attention Restoration Theory (ART) and Psychoevolutionary Theory (PET) are two theoretical frameworks that propose a mechanism for the emotional, cognitive, and physical benefits of being in natural spaces. Although these theories have not been experimentally operationalized in the context of green exercise, they provide a basis for understanding the mechanism that leads to the benefits of this activity. As such, they can be considered the theoretical underpinning for the additional benefits of physical activity in the natural environment. This paper will first review ART and PET, and then, given their tenets, recommendations for green exercise advertising will be outlined.

\section{Attention restoration theory}

Developed by Kaplan and Kaplan (11), Attention Restoration Theory postulates that there are two processes that individuals can utilize to attend to a stimulus; the selection of a process is dictated by contextual and structural characteristics of the surrounding environment. The first attentional process is referred to as 'directed attention'. Directed attention requires mental focus to attend to, either because the stimulus would not otherwise attract attention or because the individual must actively single out the desired stimulus from a variety of other distracting stimuli (12). As a result, constantly attending to stimuli using this process can lead to mental fatigue and decreased cognitive performance (12).

The second attentional process that one can utilize is 'fascination'. Fascination occurs when an individual is attending to a stimuli that is inherently attention-grabbing. Based on the passive nature of this state of attention, fascination can occur without any mental focus and thus is not energy dependent. Kaplan and Kaplan (11) characterized the natural environment as a setting in which the elements are inherently fascinating. Spending time attending through fascination allows for the restoration of the mental energy required for directed attention.

Although fascination is a necessary component for an environment to be deemed attention restoring, Kaplan and Kaplan (11) propose three other characteristics that natural environments must meet to restore attentional capacity: being away, extent, and compatibility (11). Being away describes the cognitive respite that comes from being exposed to a different environment or mindset. This can occur when an individual finds him or herself in a different environment, or when they are looking at images of a different environment. Therefore, the being away criterion is met when in a natural environment, but also when gazing out the window at a garden bed or looking at an image of a natural environment. The extent criterion is related to the features of the environment that attracts the fascination of the viewer. To meet this criterion, an environment must be rich and interesting. Consequently, a natural environment that includes a variety of components, such as water features and foliage, would satisfy the criterion of extent. The final criterion, compatibility, describes the need for the environment to match the intention of the viewer. For example, an individual that has immersed him or herself in an environment for the purposes of relaxation would expect to encounter an environment that serves that role. A dangerous looking or poorly kept environment would not meet the expectations of the viewer and therefore would not meet the compatibility criterion. This theory has been extensively researched, and a base of empirical evidence exists that suggests the natural environment significantly increases attentional and cognitive performance, both when immersed in natural environments and simply viewing images of nature (13-15).

\section{Psychoevolutionary theory}

Developed by Ulrich (16), Psychoevolutionary Theory postulates that exposure to natural spaces results in (1) an unconscious increase in positive affect (i.e., positive emotional states) and (2) a reduction in the physiological stress response. This two-part response is theorized to be grounded in human evolution, as natural environments symbolized safety, shelter, and a potential source of food in our distant past. Ulrich postulates that this response is mediated by precognitive appraisals of certain features of the environment. Therefore, only environments that contain certain characteristics promote this two-part re- 
sponse. Ulrich has proposed that there are six characteristics that are unconsciously perceived to be present or absent that mediate the internal affective and physiological response. These 'preferenda', as Ulrich refers to them, are as follows: complexity, structural properties, depth, ground surface texture, threat/tension, and deflected vistas and water (16). Complexity refers to the number of individual elements in a natural scene that contribute to the overall scene. Generally, moderate levels of complexity are received more positively than those on the higher and lower side of the spectrum. Structural properties are characteristics such as similar types of vegetation or natural content across a scene. The depth preferenda refers to the perceived size of the natural scene; when a natural scene is perceived as too dense and compact, or inversely too widespread and vast, it is less likely to be met with a preferential response. Ground surface texture is related to the walkability of an environment. If mobility is perceived as a challenge, then a preferential response is less likely to be achieved. The threat and tension preferenda is met with a positive affective response when a natural environment is perceived as safe. Examples of the deflected vista preferenda would be winding paths, rivers, or rolling hills. Deflected vistas are defined as structures that are assumed to continue out of the current range of vision, including walking paths or winding rivers. Deflected vistas foster curiosity about what other scenes may be just out of sight. In addition to deflected vistas, the presence of water (e.g., rivers, lakes, oceans) is also included in the last preferenda. Ulrich suggests that the presence of water in a scene is met with high positivity. Similar to ART, PET has been the subject of much experimental investigation. Several studies have supported this theory by demonstrating that exposure to nature (either in person or by looking at images) does indeed suppress physiological stress responses and increase positive emotion (14,17-19).

\section{Discussion}

The following two recommendations for green exercise advertising will be grounded in both of the preceding theories. They can be utilized in the design of both print and video advertisements. Recommendations cover two areas that govern message design; the first dictates the images that should be used in messages, and the second outlines what should be included in the textual (or potentially auditory for video) portion of the message.

\section{Message images}

The first recommendation is that images of natural environments should be included, and these images should meet the four characteristics necessary for attention restoration and the six preferenda necessary for positive affectivity and stress reduction $(11,16)$. As discussed earlier in this paper, the effects proposed in both ART and PET have been experimentally demonstrated when simply viewing images of nature, not only when physically immersed in that setting $(13,19)$. Therefore, by featuring natural images in print or video messages promoting green exercise, the benefits proposed by ART and PET can be harnessed and utilized to increase the effectiveness of the messages.

There are potentially two benefits associated with featuring these types of images. The first potential outcome associated with featuring natural images in green exercise messages is the effect the unconscious affective response may have on attitudes towards the message. Past research in advertising has determined that a positive emotional experience towards images in advertisements leads to positive attitudes towards the brand being advertised (20). This idea has formed the basis for a multitude of past advertising campaigns that have aimed to invoke a positive affectivity, which is subsequently paired with the brand. As such, it would follow that if green exercise advertisements could invoke a positive emotional experience, that positive affectivity would subsequently be associated with the focus of the message (green exercise). Furthermore, a positive attitude towards physical activity has been determined to be a factor leading to increased uptake and maintenance of physically active behaviours (21). Stated in another way, provoking a positive affect in the audience of a physical activity message may lead to an increased chance they will engage in that behaviour. As first proposed by Ulrich (19) and subsequently experimentally demonstrated, 
natural images that meet the six preferenda provoke a reduced stress response and a positive emotional experience. Based on that premise, Hartmann and Apaolaza (14) explored the effects of including natural images in advertisements based on ART and PET (referred to in that paper as Stress Reduction Theory). They found that emotional responses to a textual advertisement that remained constant over all conditions were significantly more positive when accompanied by pleasant natural images versus an unpleasant natural image or an urban image. Therefore, it would be expected that a natural image that is present in a green exercise message would invoke a positive emotional experience, which could then lead to positive attitudes towards green exercise itself.

The second outcome is related to improving recall of the message content. Hartmann and Apaolaza (14) also explored the effects of including natural images in advertisements on memory and recall in the same study as referenced above. Based on prior research that has found that message retention and recall is improved by both increased attention and positive emotion (22-24), they hypothesized that the attention restoration and positive affect that are provoked by viewing natural environments would improve the retention and recall of messages that are accompanied by such images. They found that participants could recall and recognize verbal or textual message elements significantly more when those messages were accompanied by images of nature in comparison to images of urban environments, people, or miscellaneous items. This research has major implications for the current discussion. By accompanying green exercise messages with images of natural environments that meet the ART and PET criteria, individuals who view these messages may experience attention restoration and positive emotions, which, as demonstrated by Hartmann and Apaolaza, may subsequently lead to better retention of the green exercise message itself.

It is important to note that, although some of the ART and PET criteria will be uniformly perceived across dif- ferent individuals, other criterion may be more subjective. For example, the deflected vistas/water preferenda is quite objective; as such it should be expected that two individuals would agree on the presence or absence of this criterion. Conversely, the fascination and compatibility criteria could be considered much more subjective. If two individuals are entering a natural environment with different expectations of that setting, or they have different operationalizations of what an interesting environment may look like, they may not agree if an environment meets the fascination or compatibility criteria. In these cases, tailoring specific natural images to the interests of the intended audience could increase the number of ART and PET criteria met.

\section{Message content}

The second recommendation is that green exercise promotional messages must not only explicitly state the benefits of physical activity in general, but also the emotional, cognitive, and physiological benefits of green exercise as postulated by ART and PET. This will potentially improve outcomes of green exercise messages in three ways, discussed below.

One framework commonly utilized when describing the process of behaviour change is the Transtheoretical Model, which is thoroughly discussed elsewhere in the literature (25-28). According to this model, motivation for behaviour change occurs when the perceived benefits of a particular action outweighs the barriers or 'cons' of changing the behavioural status quo (28). For individuals to decide to become physically active, these benefits must outweigh each individual's 'cons' of change. Based on the utilitarian pros versus cons approach to motivating change, it would be expected that increasing the number of benefits associated with physical activity would increase the likelihood that those pros would outweigh the cons of change. This phenomenon has been demonstrated experimentally (28). Progressing from the contemplation to action stage specific to physically active behaviours was mediated by an increase in the perceived pros of behaviour change. The benefits associated with being in natural environments as proposed by ART 
and PET include increased attentional capacity, improved cognitive processes, increased positive emotion, and reduced stress (14). By featuring these unique benefits of green exercise in addition to the commonly featured benefits of physical activity (e.g., reduced risk of disease, increased energy, etc.), the number of benefits of engaging in that behaviour is increased, as is the likelihood of behaviour engagement in the message viewer.

The addition of the unique benefits of green exercise in messages widens the breadth of benefits that are associated with physical activity in natural environments. For example, reducing the risk of colon cancer and restoring attentional capacity are both benefits associated with green exercise, but are quite different in nature. This means that there are benefits of this behaviour that would potentially appeal to a much wider audience than it would when only focusing on the health benefits of physical activity alone. For example, an individual who is not necessarily motivated to be physically active for health reasons but values their cognitive abilities may be more motivated to be physically active in natural spaces if a message also mentions the cognitive and attentional benefits of green exercise.

The Theory of Planned Behaviour is one of the major theories to propose factors determining behaviour (29). Within that theoretical framework, there are two categories of attitudes that contribute to behavioural intentions: instrumental and affective attitudes $(29,30)$. Instrumental attitudes are based on cognitive appraisals of a behaviour. In terms of physical activity, the health benefits and disease risk reduction of such a behaviour would be examples of an instrumental attitude. Affective attitudes are tied to the affective responses that are aroused in response to a behaviour. Recent research has started to explore which type of attitude is more predictive of behavioural intention and behaviour itself. Conner and colleagues (30) presented participants with a physical activity promotional message that focused on either instrumental or affective benefits of physical activity. Instrumental benefits included the health benefits of physical

\section{WURJ: Health and Natural Sciences Vol. 6}

activity (reduced risk of colon and breast cancer, increased bone health), and the affective benefits included the stress and depression reducing properties of physical activity. Conner et al. found that participants who viewed the affective condition had significantly higher intentions to be physically active and significantly higher physical activity levels than the instrumental and control conditions. Based on the above findings, including the unique benefits of green exercise in messages could improve message effectiveness because stress reduction and increased positive affect could increase an individual's affective attitude towards physical activity.

\section{Conclusion}

Exercising in natural settings has been found to foster more positive physical and emotional benefits than exercising in other environments (5-7). This paper has proposed two recommendations for designing green exercise messages, grounded in Attention Restoration Theory and Psychoevolutionary Theory. The first recommendation is to accompany messages with images of natural environments that meet the criteria put forth by both ART and PET. This will have two effects; first, the image could be expected to provoke a positive emotional experience in the viewer, who will then associate that positive affect with the message, which in this case is green exercise. Second, the attentional restoration that occurs when viewing the accompanying image may lead to better retention of the message which will increase the likelihood of the message being effective in the future. The second recommendation is to include the additional benefits of exercising in natural environments in the message itself. This design of the message content could be expected have three effects. First, these added benefits may increase the number of 'pros' of engaging in physical activity, which will increase the likelihood of individuals engaging in such behaviour. Second, the unique benefits of green exercise widen the scope of individuals that may be persuaded to engage in that behaviour. Third, the benefits of being in natural environments are characterised as primarily affective as opposed to instrumental, which have been demonstrated as potentially more effective in motivating behaviour. Taken together, 
the two recommendations presented in this paper could be expected to increase the effectiveness of green exercise messages in print and visual media.

\section{References}

1. Colley RC, Garriguet D, Janssen I, Craig CL, Clarke J, Tremblay MS. Physical activity of Canadian adults: Accelerometer results from the 2007-2009 Canadian health measures survey. Health Reports. 2011; 22(1):1-8.

2. Penedo FJ, Dahn JR. Exercise and well-being: a review of mental and physical health benefits associated with physical activity. Current Opinion in Psychiatry. 2005; 18:189-193.

3. Pretty J, Griffin M, Sellens M, Pretty CJ. Green exercise: complementary roles of nature, exercise and diet in physical and emotional well-being and implications for public health policy. Colchester: University of Essex CES Occassional Paper; 2003.

4. Pretty J, Peacock J, Sellens M, Griffin M. The mental and physical health outcomes of green exercise. International Journal of Environmental Health Research. 2005; 15(5):319-337.

5. Pretty J, Peacock J, Sellens M, South N, et al. Green exercise in the UK countryside: effects on health and psychological well-being, and implications for policy and planning. Journal of Environmental Planning and Managemen. 2007; 50(2):211-231.

6. Thompson Coon J, Boddy K, Stein K, Whear R, Barton J, Depledge MH. Does participating in physical activity in outdoor environments have a greater effect on physical and mental wellbeing than physical activity indoors? A systematic review. Environmental Science \& Technology. 2011; 45(5):17611772.

7. Gladwell VF, Brown DK, Wood C, Sandercock GR, Barton JL. The great outdoors: how a green exercise environment can benefit all. Extreme Physiology \& Medicine. 2013; 2(1):3-9.

8. Brown DR, Soares J, Epping JM, Lankford TJ, Wallace JS, Hopkins D, Ramsey Buchanan L, Orleans CT, Community Services Task Force. Stand-alone mass media campaigns to increase physical activity.

\section{WURJ: Health and Natural Sciences Vol. 6}

a community guide updated review. American Journal of Preventative Medicine. 2012; 43(5):551-561.

9. Kahn EB, Ramsey LT, Brownson R, et al. The effectiveness of interventions to increase physical activity: a systematic review. American Journal of Preventative Medicine. 2002; 22(4s):73-107.

10. Brawley RB, Latimer, AE. Physical activity guides for Canadians: messaging strategies, realistic expectations for change, and evaluation. Applied Physiology, Nutrition, and Metabolism. 2007; 32:S170S184.

11. Kaplan R, Kaplan S. The experience of nature: a psychological perspective. Cambridge: Cambridge University Press; 1989.

12. Kaplan S. The restorative benefits of nature: toward an integrative framework. Journal of Environmental Psychology. 1995; 15:169-182.

13. Berto R. Exposure to restorative environments helps to restore attentional capacity. Journal of Environmental Psychology. 2005; 25:249-259.

14. Hartmann P, Apaolaza V. Nature imagery in advertising: attention restoration and memory effects. International Journal of Advertising. 2013; 32(2):183210.

15. Tennessen CM, Cimprich B. Views to nature: effects on attention. Journal of Environmental Psychology. 1995; 15:77-85.

16. Ulrich RS. Aesthetic and affective response to natural environment. In: Altman I, Wohlwill JF, editors. Behavior and the Natural Environment. New York: Plenum Press; 1983. p. 85-125.

17. Ulrich RS. Visual landscapes and psychological wellbeing. Landscape Research. 1979; 4:17-23.

18. Ulrich RS. Natural versus urban scenes: some psychophysiological effects. Environment and Behavior. 1981; 13:523-556.

19. Ulrich RS, Simons RF, Losito BD, Fiorito E, Miles MA, Zelson M. Stress recovery during exposure to natural and urban environment. Journal of Environmental Psychology. 1991; 11:201-230.

20. Batra R, Ray ML. Affective responses mediating acceptance of advertising. Journal of Consumer Research. 1986; 13(2):234-249.

http://dx.doi.org/10.5206/wurjhns.2015-16.4 
21. Carron AV, Hausenblas HA, Mack D. Social influence and exercise: a meta-analysis. Journal of Sport and Exercise Psychology. 1996; 18:1-16.

22. Christianson SA, Loftus EF, Hoffman H, Loftus GR. Eye fixations and memory for emotional events. Journal of Experimental Psychology: Learning, Memory, \& Cognition. 1991; 17:693-701.

23. Rajaram S, Srinivas K, Travers S. The effects of attention on perceptual implicit memory. Memory and Cognition. 2001; 29(7):920-930.

24. Till BD, Baack DW. Recall and persuasion. Does creative advertising matter? Journal of Advertising. 2005; 34(3):47-57.

25. DiClemente CC, Prochaska JO, Fairhurst SK, Velicer WF, Velasquez MM., Rossi JS. The process of smoking cessation: an analysis of precontemplation, contemplation and preparation stages of change. Journal of Consulting and Clinical Psychology. 1991; 59:295-304.

26. Prochaska JO, DiClemente CC. Stages and processes of self-change in smoking: toward an integrative model of change. Journal of Consulting and Clinical Psychology. 1983; 51:390-395.

27. Prochaska JO, DiCLemente CC. (1992). Stages of change in the modification of problem behaviors. In: Hersen M, Eisler RM, Miller PM, editors. Progress in behavior modification. Sycamore, IL: Sycamore Press; 1992. p. 184-218.

28. Prochaska JO. Strong and weak principles for progressing from precontemplation to action on the basis of twelve problem behaviors. Health Psychology. 1994; 13(1):47-51.

29. Ajzen I. From intentions to actions: a theory of planned behavior. In: Kihl J, Beckmann J, editors. Action-control: from cognition to behavior. Heidelberg: Springer; 1985. p. 11-39.

30. Conner M, Rhodes RE, Morris B, McEachan R, Lawton R. Changing exercise through targeting affective or cognitive attitudes. Psychology and Health. 2011; 26(2):133-149. 
